\title{
Kooperation der Deutschen Gesellschaft für Orthopädie und Unfallchirurgie (DGOU) und der Orthopaedic Research Society (ORS)
}

Der seit 2015 bestehende Kooperationsvertrag zwischen der DGOU und der Orthopaedic Research Society (ORS) hat zu einer erheblichen Vertiefung der Zusammenarbeit beider Gesellschaften geführt. Diese drückt sich in einer Vielzahl unterschiedlicher Aktivitäten aus.

So beauftragte die DGOU ihre Sektion „Grundlagenforschung“ mit der Erstellung von Vergabekriterien für einen mit $2500 €$ dotierten „ORS Travel Award“, mithilfe dessen die aktive Teilnahme am Jahreskongress der ORS unterstützt und so der wissenschaftliche Austausch zwischen DGOU und ORS gefördert werden soll. In einer Preisträgersitzung im Rahmen des Deutschen Kongresses für Orthopädie und Unfallchirurgie (DKOU) 2016 wurde der „ORS Travel Award“ erstmalig vergeben. Auf Basis der Gutachterbewertung wurde Dr. Klemens Horst aus der Klinik für Unfall- und Wiederherstellungschirurgie der Uniklinik RWTH Aachen mit der Arbeit „First results of a porcine long-term model of combined trauma“ als Preisträger ermittelt. Dr. Horst konnte diese For- schungsergebnisse im März 2017 im Rahmen des Jahreskongresses der ORS in einer Präsentation vorstellen ( $\bullet$ Abb. 1). Er zeigte sich von dem dortigen wissenschaftlichen Austausch sehr beeindruckt, konnte zahlreiche neue Impulse für seine zukünftige wissenschaftliche Arbeit gewinnen und viele neue Kontakte knüpfen.

Auch während des DKOU 2017 wird eine Preisträgersitzung zur Vergabe des „ORS Travel Awards“ stattfinden. Die Vortragenden dieser Sitzung wurden aufBasis der Qualität des eingereichten Abstracts aus einer Vielzahl von Bewerbungen ausgewählt.

Neben der Sitzung zur Vergabe des „ORS Travel Awards“ finden im Rahmen des DKOU 2017 weitere grundlagenwissenschaftliche Sitzungen in Kooperation mit der ORS aber auch mit dem AO-Forschungsinstitut Davos statt. In jeder dieser englischsprachigen Veranstaltungen wird durch einen ausgewiesenen Experten der ORS oder des AO-Forschungsinstituts ein Hauptvortrag gehalten, dessen Einzelheiten im Folgenden dargestellt werden:

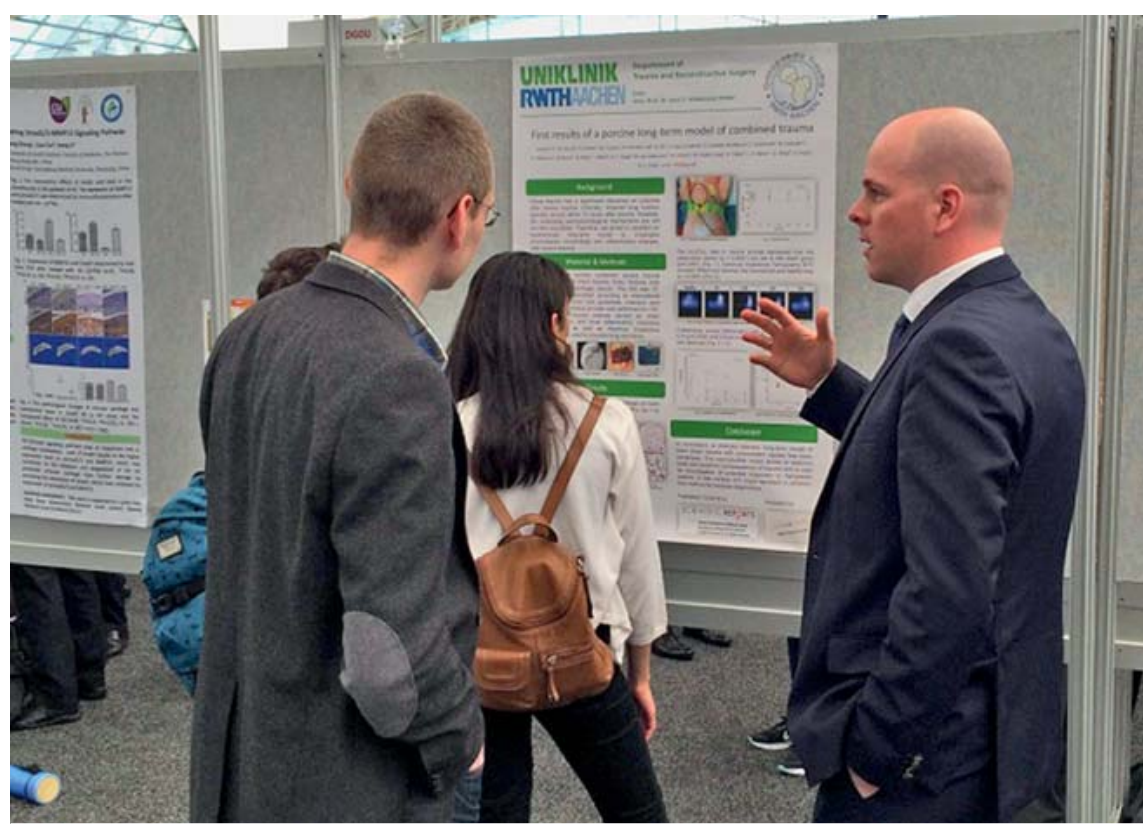

- Abb. 1 Aktive Teilnahme des Preisträgers des „ORS-Travel Awards“ 2016, Dr. Klemens Horst, am ORS-Jahreskongress 2017 in San Diego, USA.

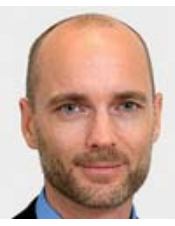

Dr. Martin Englund, PhD

Associate Professor

Clinical Epidemiology Unit, Orthopaedics

Department of Clinical

Sciences

Lund University, Sweden

„The tale of the meniscus and osteoarthritis“

Dr. Martin Englund ist aktives Mitglied der ORS und trägt regelmäßig als Gutachter und Vorsitzender zu erfolgreichen Kongressen der ORS bei. Als „Meniscus Section Officer" hat er sich in den vergangenen Jahren sehr für diese Schwerpunktbildung innerhalb der ORS eingesetzt.

Sein besonderes Interesse gilt der Bedeutung der Menisken im Rahmen der Gonarthrose. Er ist überzeugt, dass ein besseres Verständnis für traumatische und degenerative Meniskusläsionen erforderlich ist, um in der Zukunft neue Therapieansätze zu entwickeln.

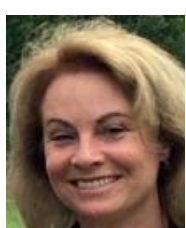

Dr. Deborah Mason, PhD Reader School of Biosciences Cardiff University, Wales Principal Investigator and Preclinical Research Program Lead

Arthritis Research UK Biomechanics and Bioengineering Centre Cardiff University, Wales „Repurposing glutamate receptor antagonists to prevent and treat osteoarthritis“

Dr. Deborah Mason ist Mitglied des „Program Committee“ der ORS und trägt als aktives Mitglied der ORS als Gutachterin und Vorsitzende zu erfolgreichen Kongressen bei. Als Vorsitzende des „Preclinical Research Program“ am „Arthritis Research UK Biomechanics and Bioengineering Centre " in Cardiff hat sie Drittmittel in Höhe von mehr als 17 Millionen Pfund eingeworben. Ihr besonderes Interesse gilt der Behandlung von Arthrose. Ihre Ansatzpunkte sind Signalwege bei degenerativ oder traumatisch eingeschränkter Gelenk- 
mechanik. Dabei treibt sie vor allem ein besseres Verständnis der biologischen Mechanismen an, die mechanische Belastung, Inflammation und Gelenkverschleiß miteinander verbinden.

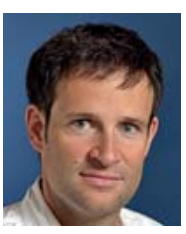

Dr. med. vet. Stephan

Zeiter, PhD, Dipl. ECLAM

Senior Project Leader

Manager Preclinical

Services

AO-Forschungsinstitut Davos

„First class science can't be done with second class preclinical surgical practice and models“

Dr. Stephan Zeiter ist „Senior Project Leader" am AO-Forschungsinstitut Davos und leitet dort die Gruppe „Preclinical Services“. Als Tierarzt ist er spezialisiert auf Labortiermedizin (Dipl. ECLAM) und leitet seit diesem Jahr als „Chair Elect" die „Preclinical Model Section“ der ORS. Sein Steckenpferd sind vorklinische Modelle mit dem Ziel, die Belastung und Anzahl der eingesetzten Versuchstiere zu vermindern und gleichzeitig die Reproduzierbarkeit und klinische Aussagekraft zu erhöhen. Er setzt sich vor allem dafür ein, dass in der Zukunft die Anzahl der verwendeten Tiere durch Alternativmethoden verringert werden kann und die verwendeten Modelle besser charakterisiert und standardisiert werden, sodass klinisch relevante Schlussfolgerungen gezogen werden können.

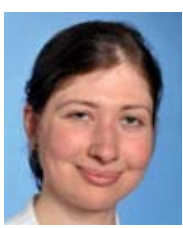

Dr. Marianna Peroglio, PhD Senior Research Scientist Musculoskeletal Regeneration

AO-Forschungsinstitut Davos

„Biobone: Bioceramics for bone repair“

Dr. Marianna Peroglio ist „Senior Research Scientist“ und Teil des „Musculoskeletal Regeneration Programs" am AO-Forschungsinstitut Davos. Dort arbeitet sie vor allem an der Biomaterialentwicklung zur Knochenregeneration und der Hydrogelapplikation im Rahmen der Bandscheibenregeneration. Sie ist der festen Überzeugung, dass In-vitro-Modelle weiter verbessert werden müssen, um die Lücke zwischen in vitro, in vivo und der klinischen Applikation schneller zu schließen. Dabei gilt ihr besonderes Interesse insbesondere Ex-vivo-Organkulturversuchen in Bioreaktoren.

Zusammenfassend lässt sich feststellen, dass sich mit den geschilderten Aktivitäten eine hervorragende Zusammenarbeit zwischen der DGOU und der ORS entwickelt hat, die auch zukünftig zu einer Internationalisierung der DGOU beitragen wird.

\section{Interessenkonflikt}

Die Autoren geben an, dass kein Interessenkonflikt besteht.

\section{Autoren}

Frank Hildebrand ${ }^{1}$, Susanne Grässel ${ }^{2}$, Ingo Marzi ${ }^{3}$, Andrea Meurer ${ }^{4}$, Michael Nerlich ${ }^{5}$, Markus Loibl ${ }^{5}$

1 Klinik für Unfall- und Wiederherstellungschirurgie, Universitätsklinikum Aachen

2 Klinik für Orthopädie, Abteilung für Experimentelle Orthopädie, Universitätsklinikum Regensburg

3 Klinik für Unfall-, Hand- u. Wiederherstellungschirurgie, Universitätsklinikum Frankfurt/ Main

4 Orthopädische Universitätsklinik Friedrichsheim gGmbH, Frankfurt am Main

5 Abteilung für Unfallchirurgie, Universitätsklinikum Regensburg

\section{Korrespondenzadresse}

\section{Prof. Frank Hildebrand}

Klinik für Unfall- und Wiederherstellungschirurgie

Universitätsklinikum Aachen

Pauwelsstraße 30

52074 Aachen

Deutschland

Tel.: 02 41/8 08-93 50, Fax: 02 41/808-2415

fhildebrand@ukaachen.de

\section{Anhang}

\section{Sitzungstermine der Sitzungen mit ORS-Beteiligung im Rahmen des DKOU 2017:}

\section{Sitzungsnummer: GR12}

Sitzungstitel: Joint Physiology and Biomechanics

Sitzungsuntertitel: In cooperation with AO Research Institute Davos

Sitzungsleitung: Bader R. (Rostock), Burgkart R. (München), Peroglio M. (Davos)

Sitzungszeit: Dienstag, 24.10.2017, 11:00-12:30 Uhr

Ort: New York 3

\section{Sitzungsnummer: GR14}

Sitzungstitel: ORS Travel Award Session

Sitzungsuntertitel: In cooperation with ORS

Sitzungsleitung: Blunk T. (Würzburg), Englund M. (Lund), Henrich D. (Frankfurt)

Sitzungszeit: Dienstag, 24.10.2017, 16:30-18:00 Uhr

Ort: New York 3

\section{Sitzungsnummer: GR16}

Sitzungstitel: Osteoarthritis and Cartilage

Sitzungsuntertitel: In cooperation with ORS

Sitzungsleitung: Grässel S. (Regensburg), Mason D. (Cardiff), Zaucke F. (Frankfurt)

Sitzungszeit: Mittwoch, 25.10.2017, 11:00-12:30 Uhr

Ort: London 2

Sitzungsnummer: GR18

Sitzungstitel: Bone Biology and Repair Sitzungsuntertitel: In cooperation with AO Research Institute Davos

Sitzungsleitung: Ignatius A. (Ulm), Schinke

T. (Hamburg), Zeiter S. (Davos)

Sitzungszeit: Mittwoch, 25.10.2017, 16:30-18:00 Uhr

Ort: London 2 